\title{
Bio-electrical impedance analysis in critically ill patients: are we ready for prime time?
}

\author{
Ivan Myatchin ${ }^{1,2} \cdot$ Paul Abraham ${ }^{3} \cdot$ Manu L.N.G. Malbrain ${ }^{1,4}$
}

Received: 21 November 2019 / Accepted: 28 November 2019 / Published online: 5 December 2019

(c) Springer Nature B.V. 2019

\section{The burden of fluid overload}

Fluid overload in critically ill patients is defined as a $10 \%$ increase in cumulative fluid balance (CFB) from baseline body weight and represents a well-known problem which detrimentally affects intensive care unit (ICU) patient's clinical course and their outcome [1-3]. Therefore, timely recognition and correction of fluid overload, or even better, identification of patients at risk for fluid accumulation in an early stage is of great importance. However, this remains challenging in clinical practice, as we miss an accurate and reliable tool for correct assessment of fluid status [4]. Current methods include the calculation of daily and CFB, by recording fluid inputs and outputs, the use of clinical or biochemical signs of fluid overload, monitoring filling pressures, however, none of these methods allow for a close monitoring for fluid balance, and intercompartmental fluid shifts $[1,5]$.

\section{The promise for BIA}

Bio-electrical impedance analysis (BIA) has gained increased interest to help physicians to determine volume status and fluid distribution in critically ill patients (see Table 1) [1, 2, 4, 6-21]. Indeed, several data published in the last decade suggest that BIA may provide useful information

Manu L.N.G. Malbrain

manu.malbrain@uzbrussel.be

1 Department Intensive Care Medicine, University Hospital Brussels (UZB), Laarbeeklaan 101, 1090 Jette, Belgium

2 Department Anaesthesiology, Sint-Trudo Regional Hospital, Sint-Truiden, Belgium

3 Anaesthesiology and Critical Care Medicine Department, Hôpital Edouard-Herriot, Lyon Cedex 03, France

4 Faculty of Medicine and Pharmacy, Vrije Universiteit Brussel (VUB), Elsene, Belgium not only in different well-established patient groups (dialysis, AIDS, malnutrition), but also in critically ill patients with burns, trauma or sepsis undergoing fluid resuscitation. This tool may offer a non-invasive, fast and reliable assessment of volume status and fluid distribution as well as evaluation of dynamic changes in fluid distribution. It measures total body water (TBW), extracellular water (ECW), and intracellular water (ICW). BIA can calculate absolute fluid overload (AFO), the difference between normal, expected ECW and the actual, measured ECW, expressed in liters, as well as relative fluid overload (RFO), AFO/ECW, expressed in percentages. BIA measurements may be performed easily at the bedside, do not require extensive training and have limited inter-observer variations [22]. Thereby, BIA sounds as a promising diagnostic tool in the ICU or operating room to assess fluid status. Recently, new BIA-devices have been introduced allowing not only calculation of TBW, ECW and ICW but also the intra- and extravascular fluids (IVF, EVF) and, in case of dialysis, Kt/V (Fig. 1).

\section{Shedding new light}

We read with great interest the work by Ciumanghel et al. [23]. Their study highlights an important clinical problem, fluid overload assessment, in a field that lacks clinical data. The study population was appropriate as abdominal surgery is among the most common elective surgical procedures [24]. The results confirm the potential role and usefulness of BIA monitoring to quantify body fluid composition and intercompartmental shifts in a major abdominal surgery perioperative setting. In this study 71 adult patients undergoing elective major abdominal surgery were included. Patients were then divided in two subgroups according to the presence of pre- and postoperative cumulative fluid overload (CFO): Normal Hydration (NH) subgroup with $\mathrm{CFO}<5 \%$, and Fluid Overload (FO) subgroup with $\mathrm{CFO}>=5 \%$. The authors found some differences between 


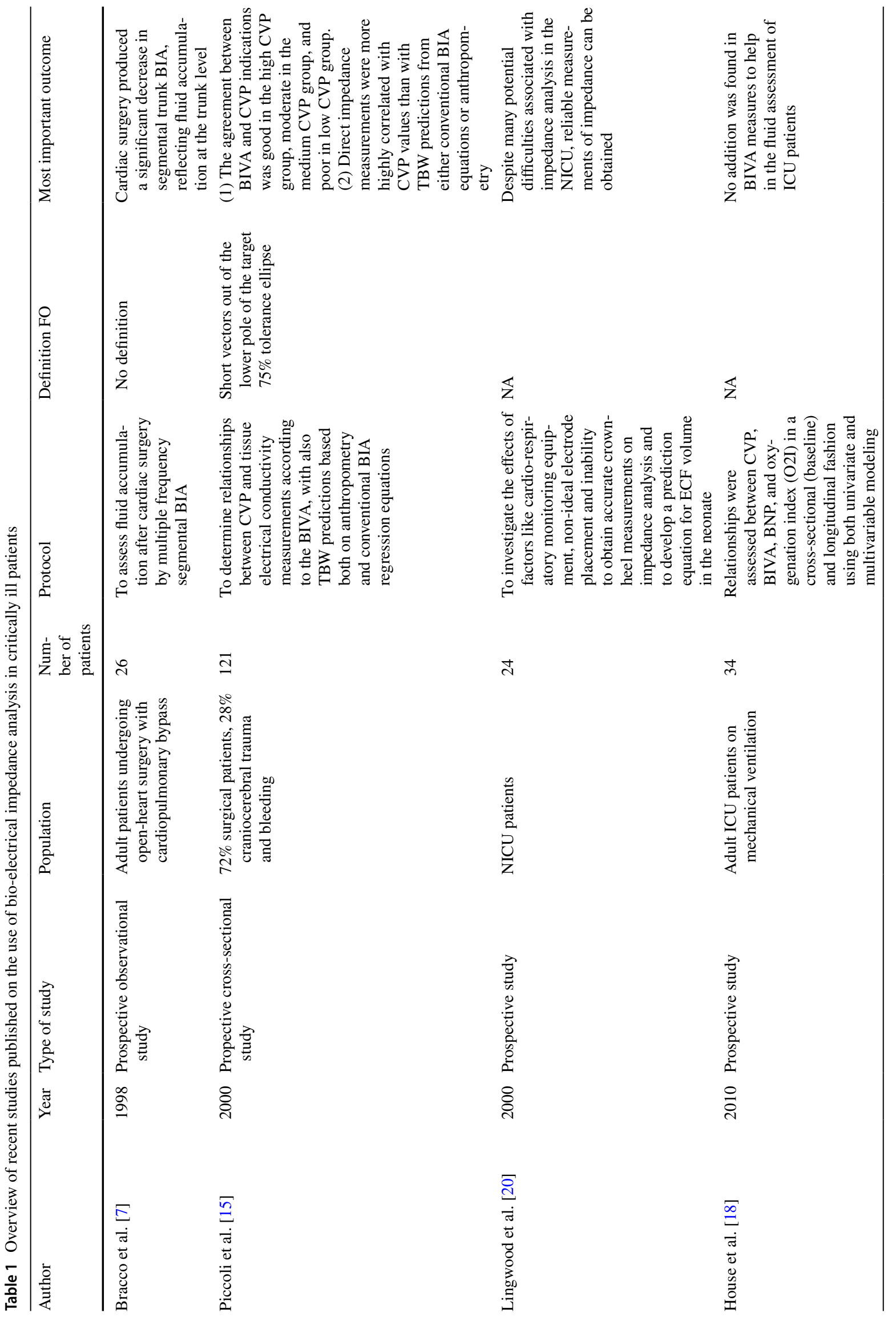




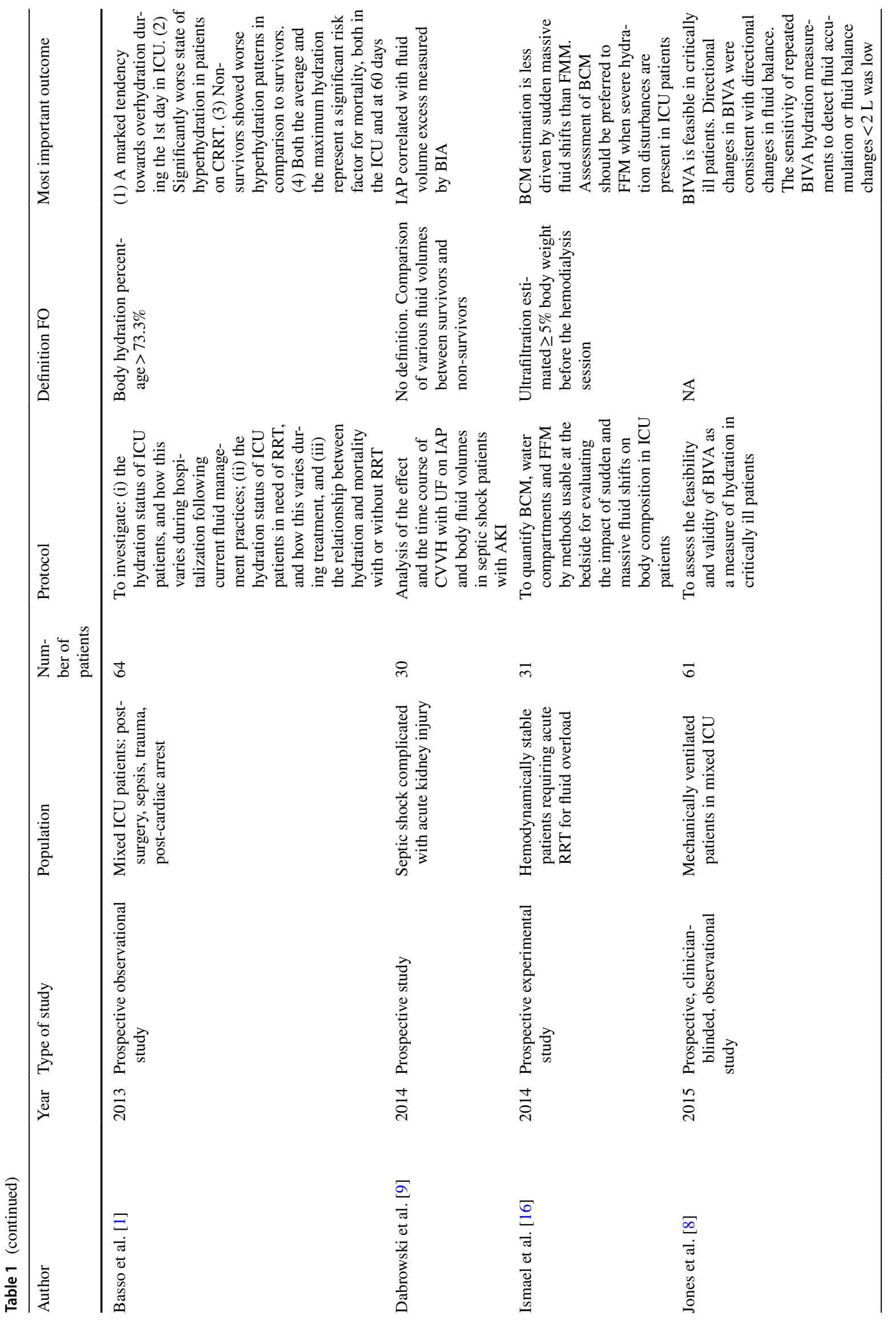




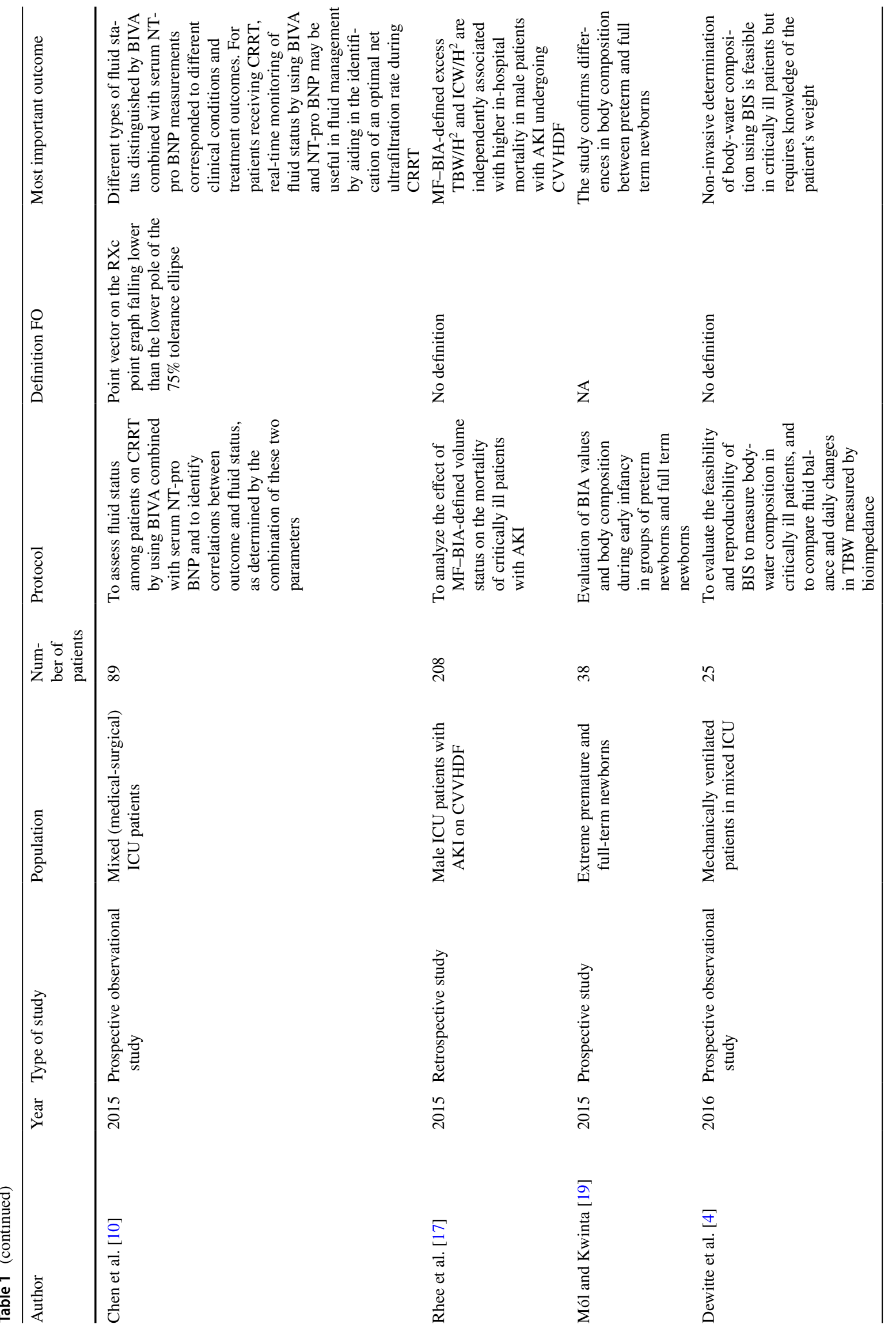




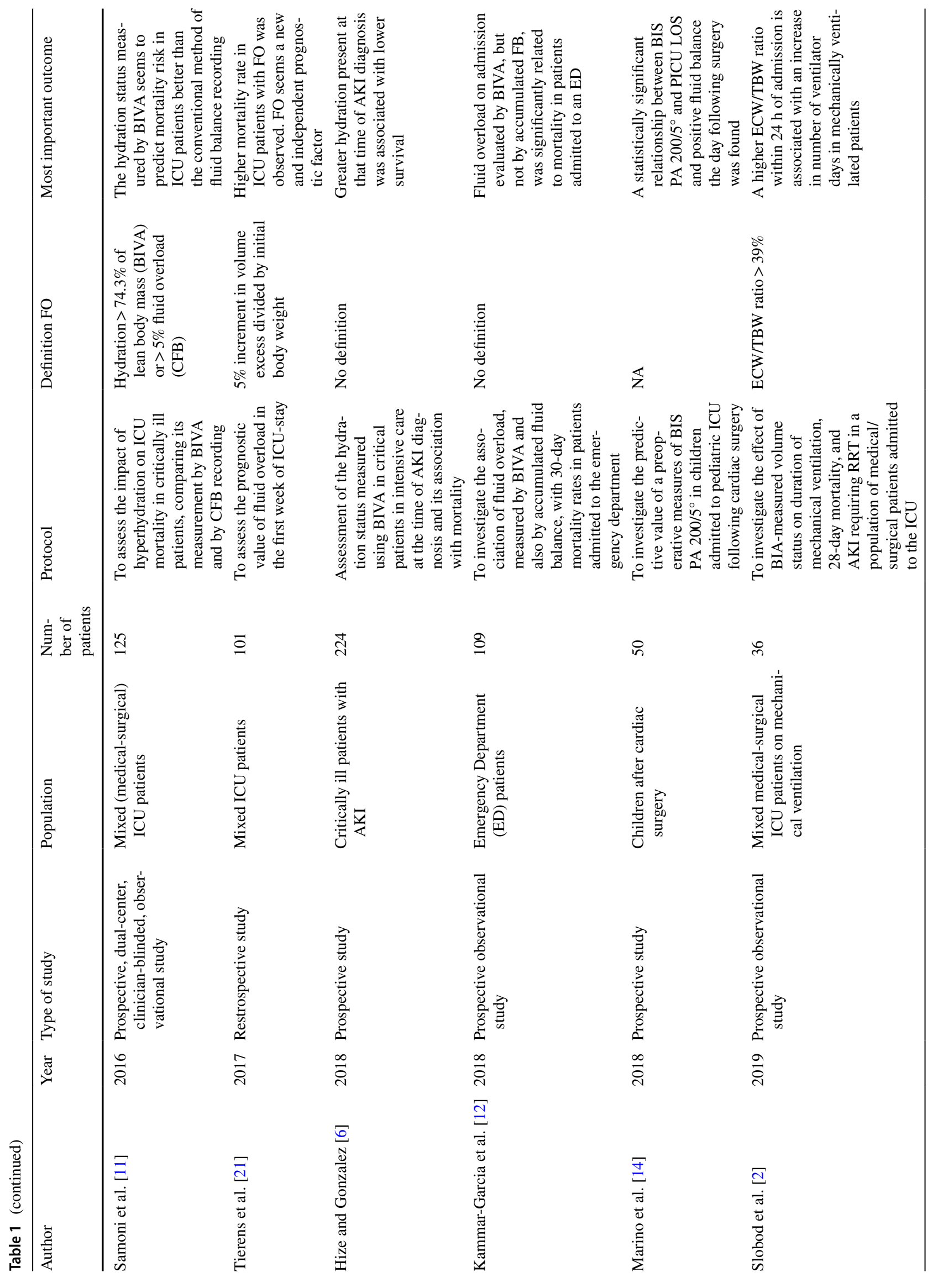




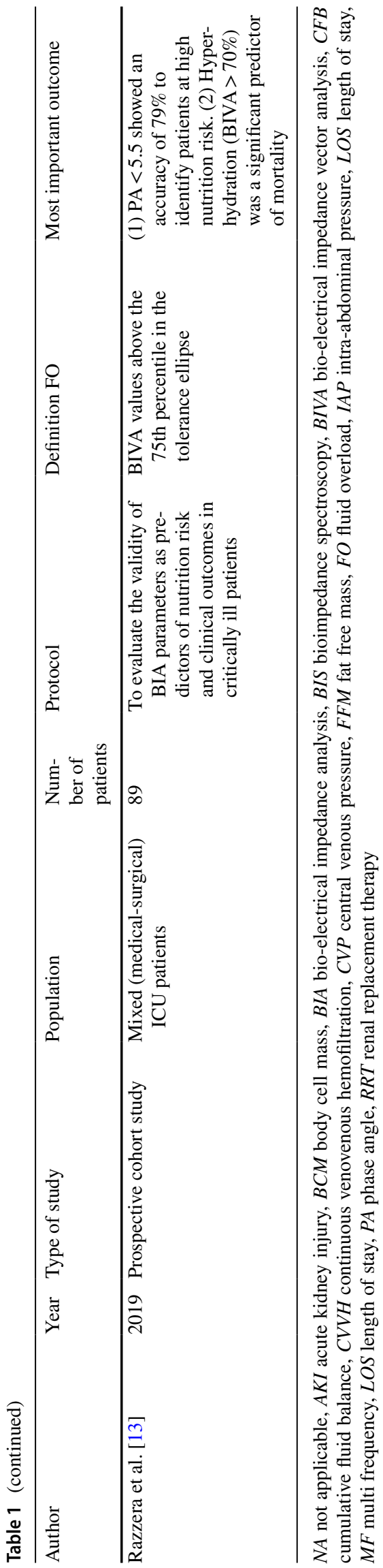

the subgroups regarding peroperative and postoperative parameters like median surgery duration and mean fluid infusion rate, amongst others. Positive intraoperative fluid balance $(2.4 \pm 1.0 \mathrm{~L})$ resulted in a significant increase of TBW $(1.4 \pm 2.4 \mathrm{~L})$ and of ECW $(1.4 \pm 1.2 \mathrm{~L})$. Intraoperative fluid balance significantly correlated with TBW change $(\mathrm{r}=0.23, \mathrm{p}=0.04)$ and with AFO change $(\mathrm{r}=0.31, \mathrm{p}<0.01)$. A significant correlation was found between pre- and postoperative AFO and RFO on one hand, and ICU-LOS on the other. In addition, we would like to provide some additional comments as food for further thought.

First of all, before surgery, patients in the FO subgroup had significantly lower haemoglobin values and significantly lower diastolic blood pressure than patients in the NH subgroup. Furthermore, patients in the FO more frequently underwent duodeno-pancreatectomy, esophagectomy and aorto-femoral bypass. This was the case in around $72.7 \%$ (16 out of 22 patients) in the FO subgroup. This form of extensive surgery usually concerns patients presenting with a serious illness like cancer or peripheral vessel disease, also having more comorbidities.

Second, postoperative serum albumin levels dropped dramatically in FO patients, while in $\mathrm{NH}$ patients it decreased to a much lesser extent. A drop of serum albumin levels in the early postoperative period is known to be multifactorial: altered metabolism, blood loss/dilution and capillary leakage-related redistribution into the third space [25-29]. The latter being probably the most important mechanism [25], which may account for $>75 \%$ of albumin decrease in the early postoperative phase. This significant parameter also appears to be related to the magnitude of postsurgical systemic inflammatory response [26, 27], which, in turn, is directly related to the extent of surgery [25] and is believed to contribute to the risk of developing postoperative complications [28, 29]. Labgaa et al. [25] identified a serum albumin decrease $\geq 10 \mathrm{~g} / \mathrm{L}$ on postoperative day 1 after elective abdominal surgery to be independently associated with a threefold increased risk for postoperative complications. Therefore, we believe that serum albumin drop in FO subgroup not only mirrors a higher intraoperative fluid regimen but may also indicates a more pronounced postsurgical systemic inflammatory response in those patients.

Altogether, those points led us to the feeling that preoperatively, FO subgroup patients may have been already more ill and therefore may have required increased fluid infusion during surgery. Indeed, even if blood loss was the same in both subgroups, FO patients received significantly more colloid infusion. Data on the inotropic and vasoactive medication use were collected but are not presented in the paper. One could expect more vasopressors in the FO subgroup. However, in order to maintain patient's blood pressure peroperatively, one can give intravenous fluid infusion or choose vasopressor drugs. Both strategies being widely 
Panel A. The new touch i8 BIA device (Maltron, UK) allows to calculate fluid excess (fluid overload, hypervolemia, hyperhydration) but also fluid underload (hypovolemia, dehydration) and body fluid separation into TBW, ECW, ICW, ECW/ICW ratio.

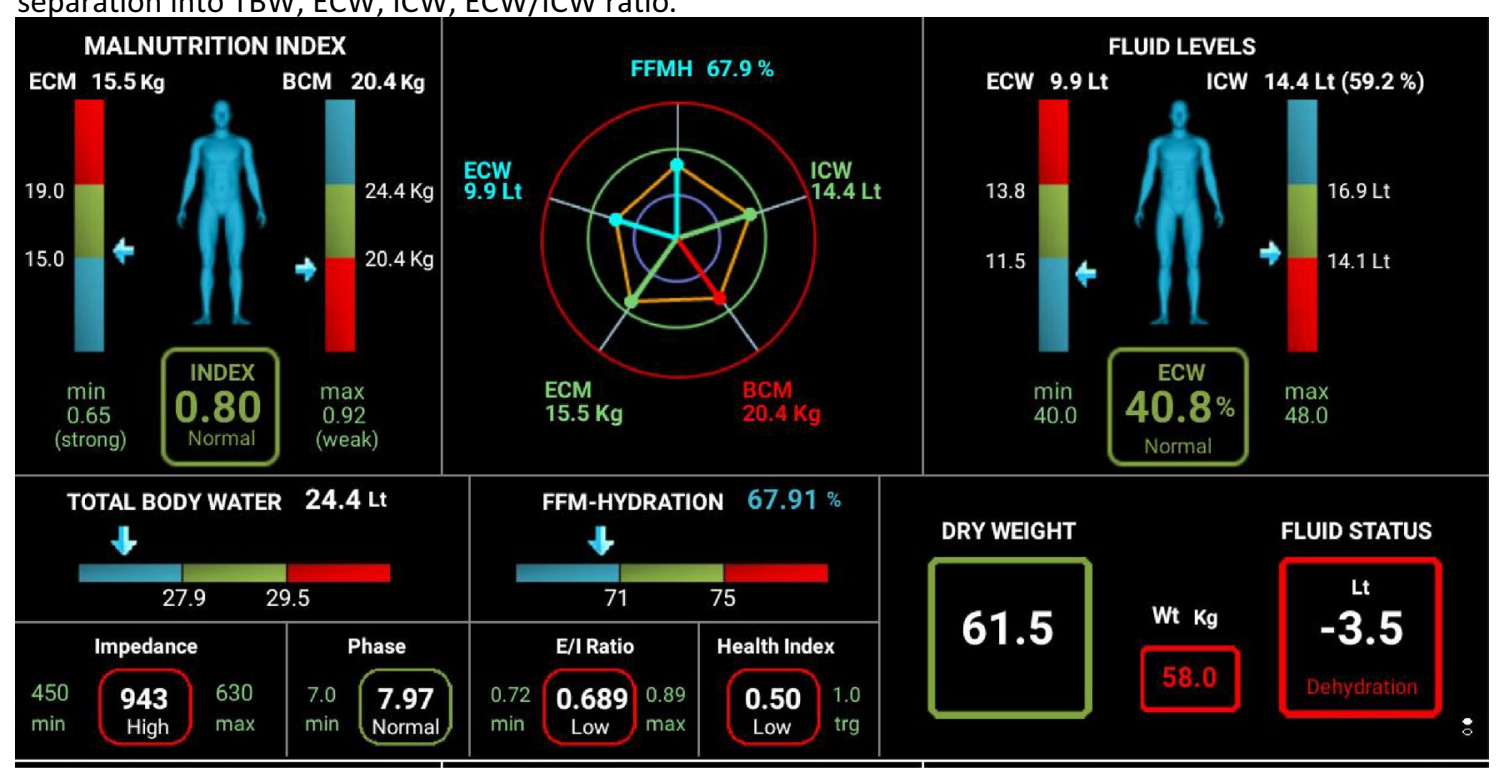

Panel B. The new software allows not only to calculate body fluid separation into TBW, ECW, ICW, ECW/ICW ratio but also IVF and EVF.

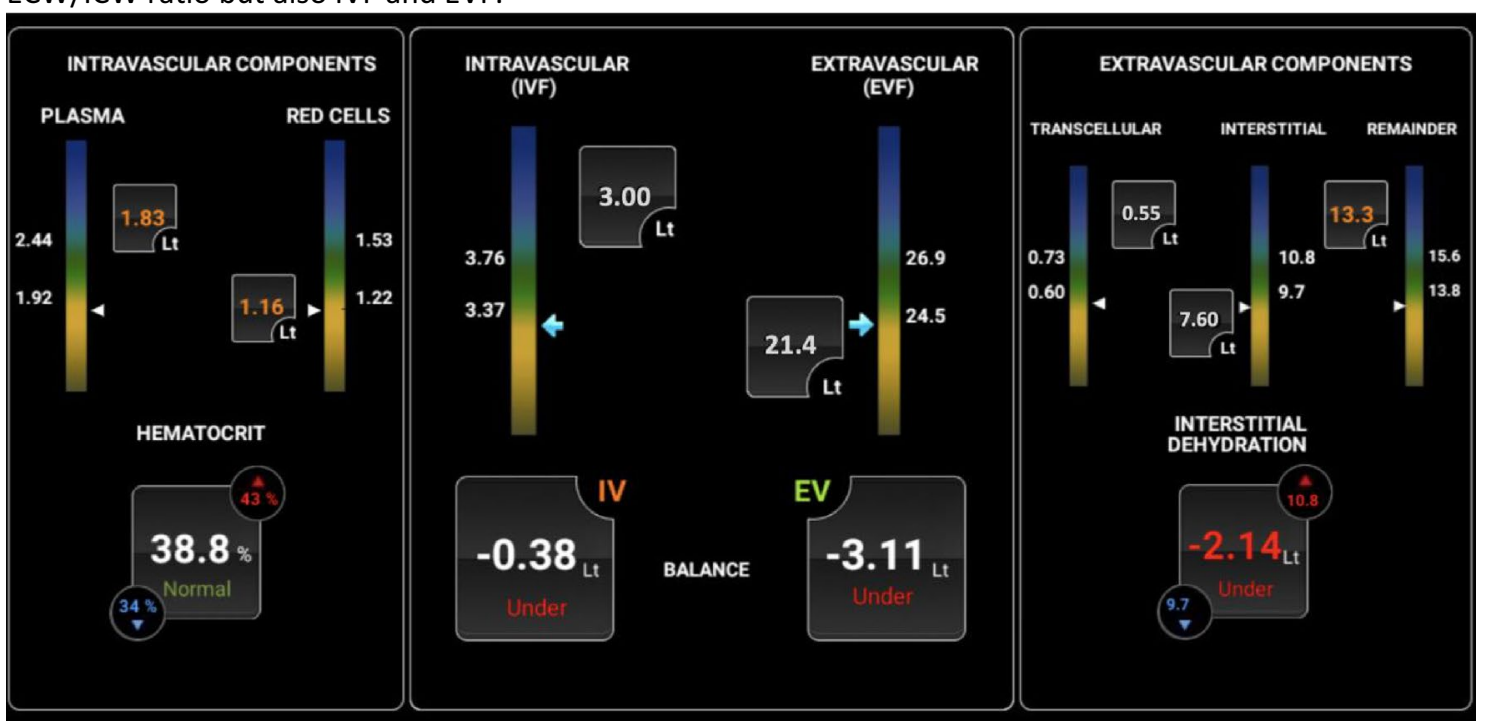

Fig. 1 The future of bio-electrical impedance analysis (BIA)

accepted in clinical practice. Unfortunately, little is known about the fluid management protocol, or whether it recommends early or late use of vasopressors? Even if fluid overload is known to impair the patient's clinical course in view of aforementioned comments, the greater rate of postoperative respiratory dysfunction and longer ICU stay in FO patients [30-32] seen in the work of Ciumanghel et al., may not be explained only by the presence of fluid overload [23].

Third, concerning BIA parameters, a comparison of pre- and postoperative BIA parameters was done for the whole group, and then in FO and $\mathrm{NH}$ subgroups separately. All these analyses consistently showed a significant increase in TBW, ECW, ECW/ICW ratio, AFO and RFO. We would also suggest a comparative analysis between $\mathrm{FO}$ and NH subgroup, as we believe that the increase in BIA-parameters in the FO subgroup would be more pronounced, due both to longer surgery and more severe illness, as partially discussed above. Moreover, the preoperative AFO and RFO values (more refined BIA parameters not related to body weight or height) were significantly 
Panel C. Evolution of BIA parameters in a male patient of 44 years with polytrauma and cumulative fluid balance of $+5 \mathrm{~L}$ on day 5, a P/F ratio 98 and EVLWI $16 \mathrm{ml} / \mathrm{kg}$ PBW. The BIA-measurement showed a volume excess of 4.9L. Initially treated with diuretics but put on CVVH with ultrafiltration afterwards. The health plot shows return to normal conditions.

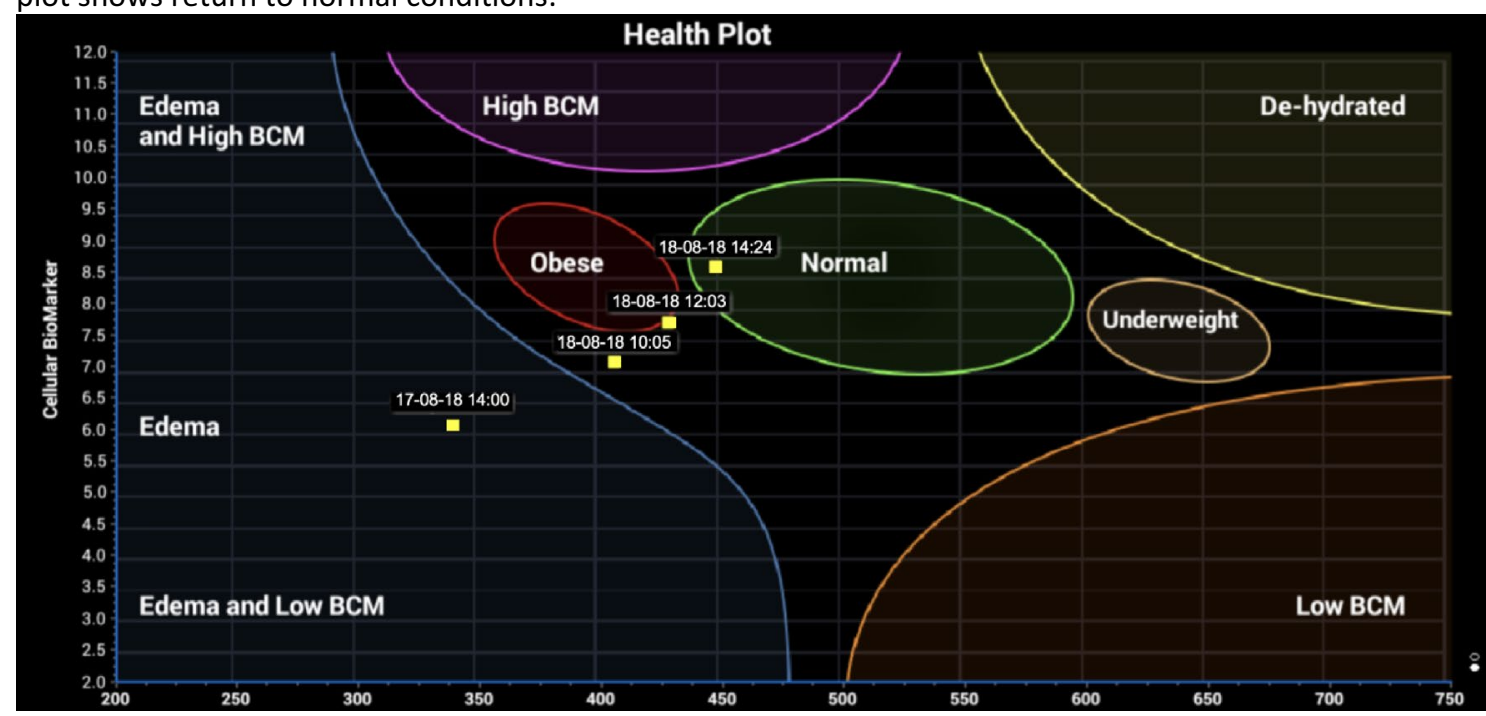

\section{Evolution of ECW and VE (in L) during fluid removal}

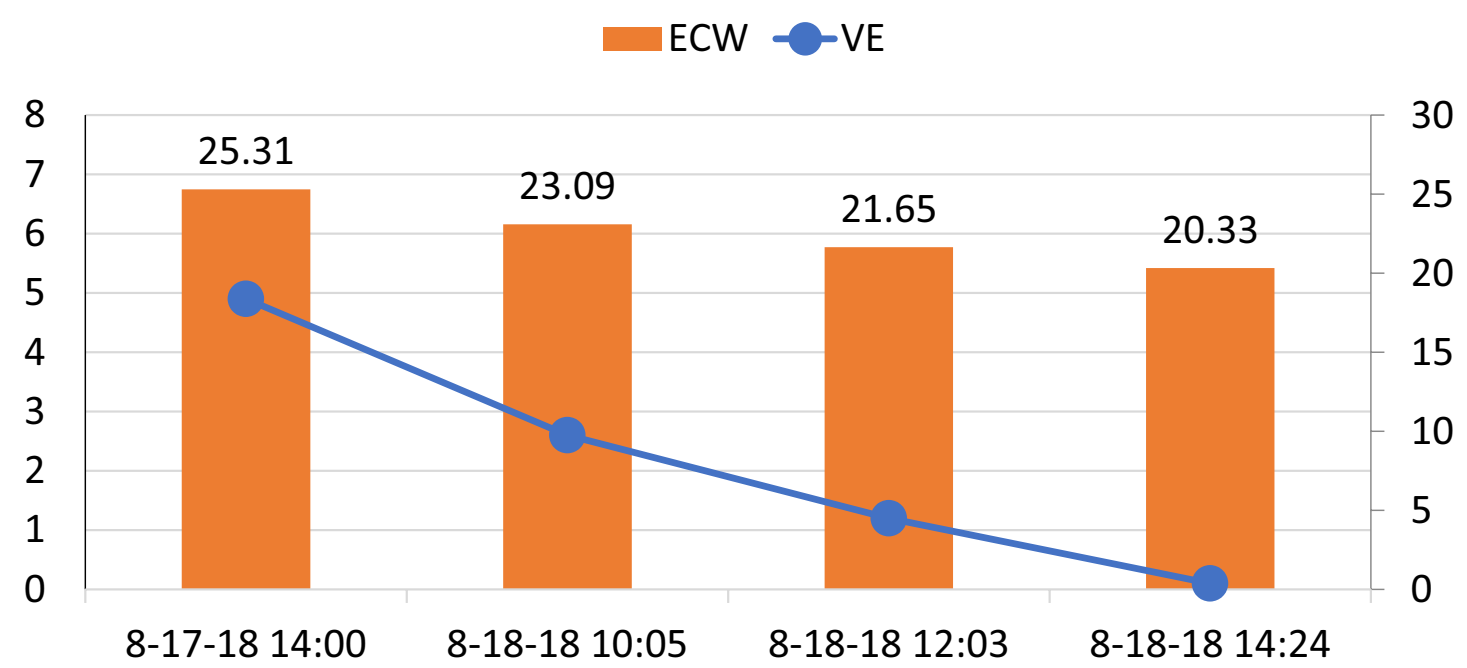

Fig. 1 (continued)

higher in FO group. This could, therefore, additionally indicate that $\mathrm{NH}$ and $\mathrm{FO}$ patients did not present with the same baseline parameters before surgery.

Fourth, regarding AFO and RFO differences between $\mathrm{FO}$ and $\mathrm{NH}$ patients, we were surprised to observe that FO patients had lower TBW and ECW values preoperatively compared to NH patients. This might be explained by significantly higher BMI values in NH patient's subgroup. Hence, it would seem appropriate to present BIA data as litre/kg body weight, as it would give a more appropriate estimation of patient's true fluid composition.

Fifth, as the authors stated themselves, having only one postoperative BIA measurement is a limitation for a broader understanding of dynamic changes in body fluid composition. More prolonged changes in the fluid balance and or distribution could probably have been revealed if later measurements would have been performed. For instance, a BIA measurement 12 and $24 \mathrm{~h}$ after ICU admission would 
Panel D. Calculation of dialysis efficiency as K.t/V after 4 hours of CVVH with ultrafiltration.

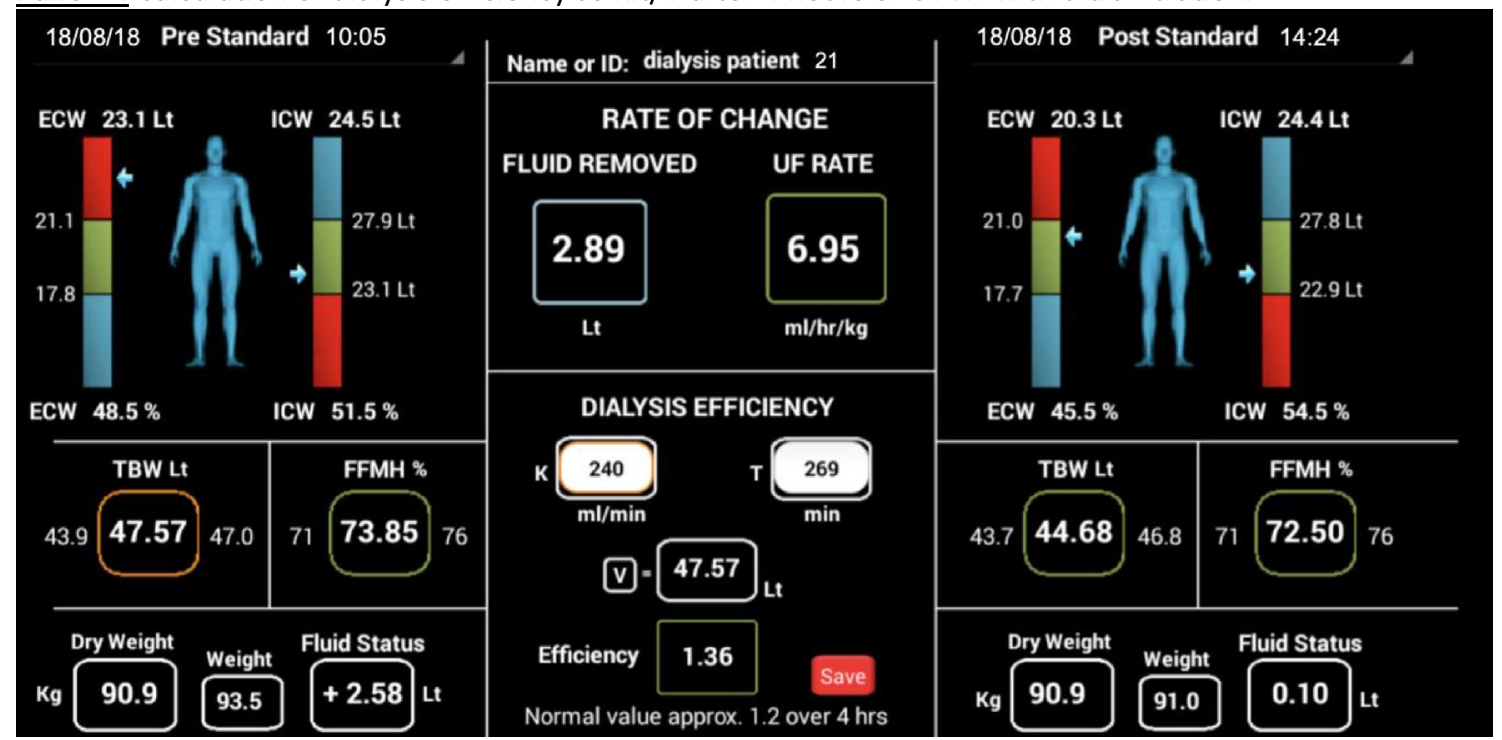

Fig. 1 (continued)

have been of great interest. Nevertheless, we recognise that this was probably challenging in the particular setting of this study, as the patients in the NH subgroup (i.e., $2 / 3$ of included patients) had a median ICU stay of $5 \mathrm{~h}$. This could be an interesting topic for further studies in the field.

\section{Take home message}

In conclusion, the study of Ciumanghel et al. addresses an important clinical problem and proposes a non-invasive, feasible, easy to perform bedside BIA-technique to assess and monitor fluid status and fluid distribution in the perioperative period, which may be of great interest to help physicians to improve management, care and outcome in critically ill patients. In the future, newer techniques may become available that allow not only calculation of TBW, ICW, ECW but also IVF and EVF. This could be of interest to assess performance of dialysis $(\mathrm{Kt} / \mathrm{V})$ but also to assess pharmacokinetics and pharmacodynamics of drugs and the fluids they are diluted in. The use of BIA in critically ill patients sounds promising but is probably not ready yet for prime time.

\section{References}

1. Basso F, Berdin G, Virzì GM, et al. Fluid management in the intensive care unit: bioelectrical impedance vector analysis as a tool to assess hydration status and optimal fluid balance in critically ill patients. Blood Purif. 2013;36(3-4):192-9.
2. Slobod D, Yao H, Mardini J, et al. Bioimpedance-measured volume overload predicts longer duration of mechanical ventilation in intensive care unit patients. Can J Anaesth. 2019;66(12):1458-63.

3. Rosenberg AL, Dechert RE, Park PK, Bartlett RH. Review of a large clinical series: association of cumulative fluid balance on outcome in acute lung injury: a retrospective review of the ARDSnet tidal volume study cohort. J Intensive Care Med. 2009;24:35-46.

4. Dewitte A, Carles P, Joannès-Boyau O, et al. Bioelectrical impedance spectroscopy to estimate fluid balance in critically ill patients. J Clin Monit Comput. 2016;30(2):227-33.

5. Mehta RL, Clark WC, Schetz M. Techniques for assessing and achieving fluid balance in acute renal failure. Curr Opin Crit Care. 2002;8(6):535-43.

6. Hise ACDR, Gonzalez MC. Assessment of hydration status using bioelectrical impedance vector analysis in critical patients with acute kidney injury. Clin Nutr. 2018;37(2):695-700.

7. Bracco D, Revelly JP, Berger MM, et al. Bedside determination of fluid accumulation after cardiac surgery using segmental bioelectrical impedance. Crit Care Med. 1998;26(6):1065-70.

8. Jones SL, Tanaka A, Eastwood GM, et al. Bioelectrical impedance vector analysis in critically ill patients: a prospective, clinicianblinded investigation. Crit Care. 2015;12(19):290.

9. Dabrowski W, Kotlinska-Hasiec E, Schneditz D, et al. Continuous veno-venous hemofiltration to adjust fluid volume excess in septic shock patients reduces intra-abdominal pressure. Clin Nephrol. 2014;82(1):41-50.

10. Chen H, Buyun W, Gong D, et al. Fluid overload at start of continuous renal replacement therapy is associated with poorer clinical condition and outcome: a prospective observational study on the combined use of bioimpedance vector analysis and serum $\mathrm{N}$-terminal pro-B-type natriuretic peptide measurement. Crit Care. 2015;19:135.

11. Samoni S, Vigo V, Reséndiz LI, et al. Impact of hyperhydration on the mortality risk in critically ill patients admitted in intensive care units: comparison between bioelectrical impedance vector analysis and cumulative fluid balance recording. Crit Care. 2016;8(20):95. 
12. Kammar-García A, Pérez-Morales Z, Castillo-Martinez L, et al. Mortality in adult patients with fluid overload evaluated by BIVA upon admission to the emergency department. Postgrad Med J. 2018;94(1113):386-91.

13. Razzera EL, Marcadenti A, Rovedder SW, et al. parameters of bioelectrical impedance are good predictors of nutrition risk, length of stay, and mortality in critically Ill patients: a prospective cohort study. J Parenter Enter Nutr. 2019. https://doi.org/10.1002/ jpen.1694.

14. Marino LV, Griksaitis MJ, Pappachan JV. Preoperative bioelectrical impedance predicts intensive care length of stay in children following cardiac surgery. Cardiol Young. 2018;28(5):779-82.

15. Piccoli A, Pittoni G, Facco E, et al. Relationship between central venous pressure and bioimpedance vector analysis in critically ill patients. Crit Care Med. 2000;28(1):132-7.

16. Ismael S, Savalle M, Trivin C, et al. The consequences of sudden fluid shifts on body composition in critically ill patients. Crit Care. 2014;18(2):R49.

17. Rhee H, Jang KS, Shin MJ, et al. Use of multifrequency bioimpedance analysis in male patients with acute kidney injury who are undergoing continuous veno-venous hemodiafiltration. PLoS ONE. 2015;10(7):e0133199.

18. House A, Haapio M, Lentini P, et al. Volume assessment in mechanically ventilated critical care patients using bioimpedance vectorial analysis, brain natriuretic peptide, and central venous pressure. Int J Nephrol. 2010;2(2011):413760.

19. Mól N, Kwinta P. Assessment of body composition using bioelectrical impedance analysis in preterm neonates receiving intensive care. Dev Period Med. 2015;19(3 Pt 1):297-304.

20. Lingwood BE, Coghlan JP, Ward LC, et al. Measurement of extracellular fluid volume in the neonate using multiple frequency bioimpedance analysis. Physiol Meas. 2000;21(2):251-62.

21. Tierens S, Noori H, Gilleman M, et al. Assessment of fluid overload in ICU patients prognostic value of bioelectrical impedance analysis. Anaesthesiol Intensive Ther. 2019; Suppl. 1:48-50 (Poster presentation (P051) at the 6th International Fluid Academy Days, November 23-25, 2017, Antwerp, Belgium).

22. Malbrain ML, Huygh J, Dabrowski W, et al. The use of bioelectrical impedance analysis (BIA) to guide fluid management, resuscitation and deresuscitation in critically ill patients: a bench-to-bedside review. Anaesthesiol Intensive Ther. 2014;46(5):381-91.

23. Ciumanghel AI, Grigoras I, Siriopol D, et al. Bio-electrical impedance analysis for perioperative fluid evaluation in open major abdominal surgery. J Clin Monit Comput. 2019. https:// doi.org/10.1007/s10877-019-00334-8.

24. Noordzij PG, Poldermans D, Schouten O, et al. Postoperative mortality in The Netherlands: a population-based analysis of surgeryspecific risk in adults. Anesthesiology. 2010;112(5):1105-15.

25. Labgaa I, Joliat GR, Kefleyesus A, et al. Is postoperative decrease of serum albumin an early predictor of complications after major abdominal surgery? A prospective cohort study in a European centre. BMJ Open. 2017;7(4):e013966.

26. Hübner M, Mantziari S, Demartines N, et al. Postoperative albumin drop is a marker for surgical stress and a predictor for clinical outcome: a pilot study. Gastroenterol Res Pract. 2016;2016:8743187.

27. Smeets HJ, Kievit J, Dulfer FT, et al. Analysis of post-operative hypalbuminaemia: a clinical study. Int Surg. 1994;79:152-7.

28. Kohl BA, Deutschman CS. The inflammatory response to surgery and trauma. Curr Opin Crit Care. 2006;12:325-32.

29. Amar D, Zhang H, Park B, et al. Inflammation and outcome after general thoracic surgery. Eur J Cardiothorac Surg. 2007;32:431-4.

30. O'Connor ME, Prowle JR. Fluid Overload. Crit Care Clin. 2015;31(4):803-21.

31. Cordemans C, De Laet I, Van Regenmortel N, et al. Fluid management in critically ill patients: the role of extravascular lung water, abdominal hypertension, capillary leak, and fluid balance. Ann Intensive Care. 2012;2(1):S1 (Suppl 1 Diagnosis and management of intra-abdominal hypertension).

32. Malbrain ML, Marik PE, Witters I, et al. Fluid overload, de-resuscitation, and outcomes in critically ill or injured patients: a systematic review with suggestions for clinical practice. Anaesthesiol Intensive Ther. 2014;46(5):361-80.

Publisher's Note Springer Nature remains neutral with regard to jurisdictional claims in published maps and institutional affiliations. 